\title{
Relaciones de género \\ y arreglos domésticos: Masculinidades cambiantes en Concepción, Chile
}

\author{
Lucía Saldaña Muñoz \\ Universidad de Concepción, Concepción, Chile. \\ Email: lusaldana@udec.cl
}

\begin{abstract}
Resumen: Este artículo aborda el estudio de las relaciones de género, los arreglos familiares y las construcciones de masculinidad en el contexto de creciente participación femenina en el mercado laboral.Se exponen las dinámicas actuales de familias de sectores medios del Concepción urbano, vinculadas a la organización del trabajo doméstico y maneras de ejercer la parentalidad. Las persistentes limitaciones experimentadas por las mujeresparadesempeñarse en el ámbito productivo llevan al cuestionamiento gradual de los roles ocupados por los varones en la esfera reproductiva.Ello requiere la articulación de políticas públicas que propicien la conciliación de vida laboral y familiar de madres y padres. Esta investigación cualitativa utiliza entrevistas semiestructuradas y grupos de discusión a padres y madres, con hijos e hijas entre 0 y 14 años. La heterogeneidad de arreglos domésticos existentes estávinculada a varones que no sólo asumen labores reproductivas, sino que representan rupturas significativas en los roles de género.
\end{abstract}

Palabras claves: Relaciones de género, arreglos domésticos, masculinidades, familias, paternidades.

\section{Gender Relations and Division of Labour: Changing Masculinities in Concepción, Chile}

\begin{abstract}
This article deals with the study of gender relations, family arrangements and constructions of masculinity in the context of increasing female participation in the labour market. The current dynamics of families in middle sectors of the urban conception, linked to the organization of domestic work and ways of exercising parentality are presented. The persistent limitations experienced by women to perform in the productive sphere lead to the gradual questioning of the roles occupied by men in the reproductive sphere. This requires the coordination of public policies that promote the reconciliation of work and family life of mothers and fathers. This qualitative research uses semi-structured interviews and focus groups with parents with children aged $0-14$. The heterogeneity of existing domestic arrangements is linked to men who not only assume reproductive tasks, but also represent significant ruptures in gender roles.
\end{abstract}

Keywords: Gender relations, domestic arrangements, masculinities, families, parenthoods. 


\title{
Relações de gênero e arranjos domésticos: Masculinidades em mudança na cidade de Concepción, Chile
}

\begin{abstract}
Resumo: Este artigo aborda o estudo das relações de gênero, arranjos familiares e construções de masculinidade no contexto da crescente participação feminina no mercado de trabalho. Expoem-se as dinâmicas atuais das famílias dos setores médios da Concepción urbana, ligadas à organização do trabalho doméstico e às formas de exercer a parentalidade. As persistentes limitações vivenciadas pelas mulheres para atuar na esfera produtiva levam ao questionamento paulatino dos papéis ocupados pelos homens na esfera reprodutiva. Isso requer a articulação de políticas públicas que favoreçam a conciliação entre trabalho e vida familiar de mães e pais. Esta pesquisa qualitativa utiliza entrevistas semiestruturadas e grupos de discussão com pais e mães, com filhos e filhas de 0 a 14 anos. A heterogeneidade dos arranjos domésticos existentes está ligada a homens que não apenas assumem tarefas reprodutivas, mas que representam rupturas significativas nos papéis de gênero.
\end{abstract} famílias, paternidades.

Palavras chave: Relações de gênero, arranjos domésticos, masculinidades,

\section{Introducción}

Cambios que se manifiestan con distintos matices al interior de la región latinoamericana, como la creciente inserción laboral femenina, el envejecimiento de la población y el aumento del número de divorcios,plantean una serie de desafíos al contexto y la composición de las familias; elloha incidido en cómo se articulan las relaciones de género en el mundo urbano, conllevando cambios en las prácticas (Olavarría, 2003 y 2005, García y Oliveira, 2011; Valdés, Caro et al. 2005; Almeras 2000). En el caso de la participación laboral femenina, aún luego de décadas de constante aumento, Chile es uno de los países en la región que presenta una de las menores tasas de participación (García y Oliveira, 2011), persistiendo de este modola conformación de mercados laborales como "gendered institutions" (Elson, 1999), dado que en ellas ocurre la intersección entre la economía productiva y la reproductiva. De acuerdo a Elson (1999, p. 612), "la mayoría de las instituciones en el mercado laboral se construyen sobre la base de que las cargas de la economía reproductiva serán y deberían ser, asumidas mayormente por las mujeres”.

En línea con lo planteado por Elson (1999), en un artículo ampliamente citado, Acker's (1990) citado en Ranson (2012, p. 743) sostiene que diversas investigaciones documentan hasta qué punto las instituciones están influenciadas por las diferencias de género, y cómo la expectativa general sobre el "trabajador ideal” es que su vida familiar no interfiera en su ámbito laboral, lo que incide en las expectativas sociales en relación al tiempo dedicado al trabajo y a la familia por parte de los hombres, las que son muy inferiores a las esperadas para las mujeres -como se observa en las prácticas de participación masculina en la vida reproductivaen la región. 
En términos generales, la concepción tradicional del equilibrio trabajo-familia se ha basado generalmente en una marcada diferenciación sobre las expectativas en los roles de las madres y los padres; mientras que el trabajo se ha visto como la "normalidad de lo que hacen los padres", trabajo remunerado y maternidad se definen como ámbitos opuestos (Ranson; 2012, p. 741). El cambio de expectativas en relación al rol de los padres, ha llevado a reconceptualizar las políticas trabajo-familia y los programas sociales en términos "neutrales" de género en determinados contextos sociales, como es el caso de Inglaterra. (Ranson; 2012, p. 741) Así, el resultado de ello es que las madres terminan siendo las beneficiarias de programas orientados a la familia, lo que refleja que las prácticas laborales realizadas por los hombres, aún requieren recorrer un camino que logre equilibrar las responsabilidades familiares (Ranson; 2012, p. 742).

Esto ocurre en un contexto social en que existen significativos avances en equidad de género, mientras que en el caso de Chile los desafíos son aún mayores. En los nuevos arreglos que se intentan articular, Ranson (2012) plantea que se echa de menos la presencia de "padres trabajadores" -hombres que aprovechen las iniciativas generadas en el lugar de trabajo que comúnmente usan las mujeres, para articular y organizar sus vidas laborales acorde a las responsabilidades familiares que se comprometen a asumir. Hay ciertos intentos incipientes de estos en otros contextos sociales (Brandth y Kvande, 2002).

Ello se evidencia en estrategias complejas de conciliación, en particular para los padres (Ranson, 2012), dificultando las posibilidades que estos ejerzan un rol paternal más activo (Rehel, 2014). En el contexto latinoamericano, García y de Oliveira (2011) y Faur (2006), han relevado las limitaciones de las políticas de conciliación existentes, las que finalmente disminuyen las posibilidades de involucramiento paternal.Los hallazgos de la investigación destacada de Doucet (2004), quien indagó en los discursos sobre paternidad de 120 padres canadienses que realizaban el rol de cuidadores de sus hijos/as, evidencian que ellos cumplían incluso el rol de cuidadores emocionales. En ello fue central el papel ejercido por las madres, de manera que los varones asumieran responsabilidad emocional por sus hijos/as.

En particular, en el ámbito de la corresponsabilidad, tomamos como referencia el trabajo de Gómez y Jiménez (2015), quienes plantean que ésta implica la percepción de una responsabilidad compartida, así como actuar de manera coordinada como familia, teniendo en consideración dimensiones como el trabajo doméstico, el cuidado de menores y ancianos, y el trabajo emocional. Por tanto, el debate sobre conciliación requiere pensar en “conciliación con co-responsabilidad”. (Gómez y Jiménez; 2015, p.391)

En el caso de esta investigación, se analiza la realidad chilena urbana (Olavarría 2003, 2005; Valdés 2009; Valdés, Caro et al. 2005); en particular,los estudios realizados en sectores medios profesionales muestran que han tenido mayor acceso a conocer realidades diversas caracterizadas por una 
mayor igualdad de género (Valdés, Caro et al. 2005; Campos y Saldaña, 2018). Ello se relaciona con una gradual rearticulación de las relaciones de género en el hogar, incorporando mayores niveles de participación de ambos integrantes de la pareja en la definición de tareas y responsabilidades domésticas, así como también en los mecanismos de negociación de estos arreglos; incluso en algunos casos,se produce una reasignación de roles tradicionales de género, mostrandola pluralidad de masculinidades existentes.

Al mismo tiempo, un ámbito que articularelaciones de género y construcciones identitarias masculinas y femeninas, se refiere a la inserción formal en el mercado del trabajo, dado que la vida laboral ha pasado a tener un rol significativo en las definiciones identitarias personales. En particular, se ha estudiado el rol de las transformaciones del mercado laboral en las identidades masculinas (McDowell, 2004; Díaz, Godoy et al. 2005; Arango, León et al. 1995). El concepto de masculinidades ha sido extensamente abordado por R. Connell (Connell 2005; Connell y Masserschmidt, 2005;Carrigan, Connell et al. 1985); en particular, en su propuesta conceptual de masculinidad hegemónica entendida como "la configuración de práctica genérica que encarna la respuesta corrientemente aceptada al problema de la legitimidad delpatriarcado, la que garantiza (o se toma para garantizar) la posición dominante de los hombres y la subordinación de las mujeres” (Connell, 1995, p. 39). Para Connell, lo central en esta conceptualización es que la definición de la masculinidad requiere

“...centrarnos en los procesos y en las relaciones a través de los cuales los hombres y las mujeres viven vidas ligados al género. La masculinidad, hasta el punto en que el término puede definirse, es un lugar en las relaciones de género, en las prácticas a través de las cuales los hombres y las mujeres ocupan ese espacio en el género, y en los efectos de dichas prácticas en la experiencia corporal, la personalidad y la cultura (Connell; 2003, pp.108-109)

De esta forma, las construcciones de masculinidad actuales, emergen en el contexto de cambiosque están ocurriendo en la interacción entre la esfera laboral y la familiar, redefiniéndose así los roles de género al interior de la sociedad chilena, en especial en las grandes urbes. De acuerdo al informe OIT-PNUD (2009, p. 13), ello contribuye a la generación de grandes tensiones, vinculadas a nuevas formas de organización del trabajo -evidenciadas en su flexibilización, incertidumbre, auto explotación vulnerabilidad, entre otros (Saldaña, 2009). Según OIT-PNUD (2009), ello tiene un gran impacto en las mujeres, dado que se agudizan las desigualdades socioeconómicas y de género, y se dificulta la proveeduría de“ingresos seguros” en trabajos no atípicos (Craig, Powell et al. ,2012).

Lo anterior plantea desafíos en términos de políticas públicas, ya que la "conciliación entre vida laboral, familiar y personal y la corresponsabilidad social en las tareas de cuidado... formen parte de la 'corriente principal' de políticas y programas sociales de los gobiernos” 
(OIT-PNUD, 2009, p. 17), llevando a vincular trabajo decente con igualdad de género, modificando asíla división sexual del trabajo. La igualdad de oportunidades de acceso al mercado laboral releva la necesidad de su correlato en arreglos domésticos participativos y corresponsables -uno de los ejes centrales en el debate sobre igualdad de género, más aún en el contexto en que muchos hogares se ha consolidado la doble proveeduría.

En su estudio sobre relaciones de género, Coltrane (2010), analiza cómo los postulados teóricos de género inciden en los arreglos domésticos, en tanto los roles sexuales dan cuenta de una "organización de la reproducción definida por género" -que está crecientemente cuestionándose. En una línea similar, Ridgeway y Correll (2004) plantean el impacto de creencias hegemónicas sobre género y la persistencia de su jerarquización en distintos ámbitos. Ello evidencia la naturalización de la dominación masculina, extensamente discutida por Bourdieu (2000).

En relación a las identidades de género de los varones, el PNUD (2010) plantea que éstos "se resisten y limitan el acceso de las mujeres a los puestos de poder en distintos ámbitos de la conducción social; por otra, se restan a participar igualitariamente en las tareas del ámbito doméstico"(PNUD; 2010, p. 92), visibilizando los “privilegios” masculinos vinculados a las representaciones tradicionales de las relaciones de género,constructos que otorganpoder en distintos ámbitos y ventajas sólo por la condición de género (PNUD, 2010).Se observa que se va resquebrajando la "imagen tradicional de masculinidad es la idea de que los hombres, por sus capacidades, por su fuerza, por su poder, y por su autocontrol racional pueden y deben ordenar el mundo de los demás: mujeres, niños, débiles e irracionales” (Seidler 1994, 1997 citado en PNUD, 2010, p. 93).

Esa fisura se evidencia en algunas reediciones de modelo tradicional en la organización actual del trabajo doméstico: una parte sustancial de los varones continúanasumiendo labores como losarreglos del hogar, y algunas tareas cotidianas a partir de orientación femenina (Campos y Saldaña, 2018); sin embargo, se participa crecientemente en el cuidado y la crianza de hijos e hijas.Como sostiene José Olavarría (2003, p. 348), se está experimentando "un proceso de transformaciones profundas en la configuración de familias y paternidad", a la vez que estaría poniendo a prueba "la familia patriarcal nuclear”, tanto en su significado subjetivo como en relación a la práctica misma de la paternidad. Olavarría(2000, p.11) también plantea que "es posible identificar una cierta versión de masculinidad que se erige en "norma” y deviene en "hegemónica", incorporándose en la subjetividad tanto de hombres como de mujeres, que forma parte de la identidad de los varones y que busca regular las relaciones genéricas”. Es precisamente ese tipo de masculinidad la que permite reeditar el modelo tradicional a partir de una participación acotada en la vida doméstica.

La participación parental expresa uno de los principales cambios en la articulación de los arreglos domésticos y en la definición de roles en la vida reproductiva. ParaValdés, Caro et al. (2005, p. 206), se va instalando un 
carácter más democrático en las relaciones de género, con ejes centrales como "la transformación de la paternidad incorporando "la preocupación por el niño, con una dedicación variable a él”.En algunos casos, incluso se observa la herencia de patrones igualitarios de padres que han superado la imagen de la familia convencional,quienes estarían más expuestos a experiencias multiculturales, tendiendo a incorporar diferentes formas de vida. En todas las clases sociales estudiadas, se refleja cómo la incorporación de la mujer al trabajo remunerado pasa a ser un gatillador de cambios, no obstante aún sean insuficientes los niveles de democratización en las parejas y en la articulación de nuevas formas de parentalidad, dado que la identidad femenina seguiríamarcada exclusivamente por la maternidad(Valdés, Caro et al.; 2005, p. 210). Este hallazgo es un punto de partida significativo para investigaciones recientes, dado que aparecen con claridad cambios en las formas de asumir la maternidad y paternidad, por lo que es necesario conocer hasta qué punto esas formas se están modificando y qué identidades femeninas y masculinas emergen. Hay estudios que indican que existen identidades femeninas definidas por su identidad laboral y no sólo por su identidad como madres (Todaro y Yañez, 2004), como también la evidencia sugiere que la identidad masculina está crecientemente permeada por su paternidad (Ranson, 2012; Brandth y Kvande; 2002).

En esta línea, Valdés, Caro et al (2005, p. 211) sostienen que han cambiado las concepciones y el modo de construir familias, permitiendo la mayor afirmación del sujeto en tanto individuo. No obstante la madre trabaja, sigue articulando la mayoría de los ámbitos de la vida familiar, y esto dificultaría la redefinición de los roles de género (Campos y Saldaña, 2018). Es precisamente el mayor involucramiento en la parentalidad, lo que permite reformular el lugar que toma el padre en la vida familiar, quien "elige" tener cada vez una mayor participación.

En síntesis, se observa la coexistencia depatrones familiares tradicionales con otros modernos, que han conseguido romper de manera significativa los modelos más conservadores heredados, en algunos casos reeditándolos. Ello se evidencia en la heterogeneidad de arreglos domésticos y en la pluralidad de los modelos paternales existentes, lo que se indaga en esta investigación en el contexto del Concepción urbano.

Para contextualizar el caso empírico que se expone en este artículo, se presentan a continuación algunos antecedentes referidos a la inserción laboral femenina y a la carga de trabajo reproductivo en los hogares chilenos, y de la región del Bío Bío en particular.

La Primera Encuesta Nacional sobre Uso de Tiempo en Chile (Instituto Nacional de Estadísticas, 2016) da cuenta de las diferencias en la carga global de trabajo entre mujeres y hombres:considerando trabajo remunerado, no remunerado, de cuidados y de traslado, las primeras dedican 10,54 horas a la semana y 8,64 los segundos. Al desagregar los datos, las mujeres dedican, en promedio, 5,89 horas al trabajo no remunerado, más del doble que los varones $(2,74)$, brecha que se mantiene al desagregar por día de 
semana o de fin de semana (Instituto Nacional de Estadísticas, 2016).De acuerdo al INE (ENE 2017 y EME 2017), el 48,5\% de las mujeres participa del mercado laboral.

En particular, en la región del Bío-bío, la brecha de género respecto al trabajo no remunerado es similar, aun cuando las horas destinadas a éste sean menores que el promedio nacional, siendo de 2,68 para los hombres y 5,75 para las mujeres (Instituto Nacional de Estadísticas, 2016). En relación al trabajo de cuidados no remunerado, también existen importantes diferencias, donde las mujeres dedican 2,87 horas a éste versus 1,46 de los hombres -tendencia también observada en el promedio nacional. Respecto a los tiempos destinados al trabajo remunerado, traslados y la participación en el mismo al interior de la región, sumado a los tiempos de traslado, los hombres dedican 7,29 horas con una participación de 83,2\%, versus 6,11 horas que dedican las mujeres, con una participación de 80,8\% en la región (Instituto Nacional de Estadísticas, 2016).

Por otro lado, la encuesta Internacional sobre Masculinidades y Equidad de Género IMAGES, realizada en Chile el año 2011, evidencia que son los varones más jóvenes quienes presentarían actitudes más orientadas hacia la equidad de género de acuerdo a la escala de actitudes de género GEM, así como una mayor participación en las labores de cuidados y en la realización de tareas domésticas (Aguayo, Correa y Cristi, 2011).

Las tensiones respecto de la conciliación trabajo familia existentes en nuestro país y la persistencia de desigualdades de género son otros de los aspectos relevados por el estudio (Aguayo, Correa y Cristi, 2011) Ello hace particularmente importante abordar lasformas en que se estarían redefiniendo las relaciones de género y la heterogeneidad de los arreglos domésticos presentesen el Concepción urbano, permitiendo aportar a visibilizar los desafíos en materia de políticas de conciliación y de simetría en las relaciones de género al interior de los hogares.

\section{Método}

Esta es una investigación cualitativa y descriptiva. La unidad de análisis son los discursos de parejas con hijos e hijas entre 0-14 años del Concepción urbano, en que ambos integrantes desempeñan trabajo remunerado fuera del hogar, y provienen de sectores medios. El rengo etario de los y las menores se determinó a partir delas exigencias adicionales que esta etapa del ciclo de la vida pone en la pareja en términos del trabajo reproductivo, dada su mayordependencia y la mayor exigencia de dedicación de tiempode sus cuidadores, claramente reflejada en la organización de la vida familiar. Se determinó realizar este estudio en el área urbana de Concepción por la importancia y especificidad que tienen los sectores urbanos en la redefinición de las relaciones de género al interior de la sociedad chilena.Por sectores medios, se entienden profesionales de rangos medios, subgerentes, técnicos, medianos empresarios, trabajadores similares, entre 
otros, donde al menos un miembro de la pareja tenía alguna de las ocupaciones descritas.

El levantamiento de información se llevó a cabo a partir de la utilización de técnicas cualitativas de investigación, realizándose cuatrogrupos de discusión,una entrevista grupal y 112 entrevistas semiestructuradas individuales. La muestra está compuesta por 129 informantes: 82 padres y 47 madres, de las cuales 20 entrevistas fueron realizadas a ambos integrantes de la pareja por separado.

Elmuestreo fue de tipo intencionado opinático; las principales estrategias de acceso a informantes fueron el reclutamiento por medio de colegios y jardines infantiles, y la bola de nieve. Los criterios definidos fueron: sexo, tipo de vinculación laboral, nivel educacional, etapa del ciclo de la vida, y adscripción de clase.Toda la información fue grabada y posteriormente transcrita. El diseño de análisis textual utilizado fue el análisis de contenido, apoyado en el software atlas-ti 7.5.2.

\section{Resultados}

En esta sección, se exponen los principales hallazgos de la investigación que permiten caracterizar las relaciones de género, los arreglos domésticos y su incidencia en las masculinidades de Concepción urbano.

\section{Arreglos familiares diversos: el trabajo como articulador de los tiempos}

Un primer hallazgo en las familias estudiadas, es que el trabajo emerge como un eje articulador de los tiempos en la vida de las personas. Ello es particularmente visible en el caso de las mujeres, quienes asumen la responsabilización por las tareas domésticas, independientemente de su vinculación laboral parcial o completa; esto se observa enuna alta exigencia y mayor necesidad de conciliación entre tiempos de trabajo productivo y reproductivo, conllevando el desafío de delegar y compartir al menos algunas tareas y responsabilidades domésticas.

En el ámbito subjetivo, el trabajo realizado fuera del hogar cobra gran relevancia para las mujeres que trabajan jornada completa. Se observa en ellasuna búsqueda de espacio personal, más allá de la rutina doméstica y laboral; el trabajoremunerado tiene un significado vinculado a la realización personal, y es generalmente respaldado por sus parejas, como se observa en el siguiente caso:

“... si nosotros estamos bien, haciendo algo que nos gusta, vamos a estar bien también para nuestro hijo, podría decirse que tiene una... una, una buena repercusión de que nosotros trabajemos”. (Braulio, 30 años) 
El reconocimiento de los varones respecto a la importancia del trabajo femenino,podría contribuir a su creciente participación en las labores domésticas y de crianza; no obstante lo anterior,de los discursos de nuestras entrevistadas y participantes de los grupos de discusión, se desprende que son ellas quienes se encuentran muy exigidas en los esfuerzos por compatibilizar trabajo productivo y reproductivo, tendencia que se ha señalado en la literatura (PNUD, 2010; Elson, 1999; Gómez y Jiménez, 2015). En el caso de las participantes dela técnica grupal, llama la atención que el trabajo no se conceptualice como sobrecarga, sino más bien como exigencia y limitaciones a los tiempos personales, los que en algunos casos son utilizados para realizar estudios, mejorandoasí su calificación y oportunidades laborales.

\section{Liderazgo femenino: reedición de la responsabilización}

El desafío de compatibilizar trabajo productivo y reproductivo no solo plantea una exigencia en términos de los tiempos de madres trabajadoras, sino que también está fuertemente ligado al liderazgo que ejercen las mujeres en la organización de la vida doméstica familiar, muchas veces guiando al hombre en las labores y responsabilidades que requieren ser compartidas.El liderazgo femenino se expresa en la flexibilidad que las madres trabajadoras buscan conseguir para conciliar vida laboral y vida familiar. Un entrevistado da cuenta de los requerimientos de esta articulación con su pareja:

"Si bien en un principio fue más complicado, ahora no es tanto porque ehh la mamá de mi hija igual tiene jornadas laborales relativamente flexibles porque ella hace clases en una, en una universidad. Entonces los horarios en que obligatoriamente tiene que estar, son los horarios de clases, el resto del tiempo se puede ir moviendo" (Ricardo, 32 años) sostiene:

En relación al desempeño de su propio rol, una de las entrevistadas

“... Yo soy la que guío (se ríe). Yo le digo a los niños: tienen que hacer tal cosa, o a mi esposo: "sabes hay que.... no sé... arreglar algo" [...]No , no hacemos lo mismo, no, yo siento que tengo un poco más de responsabilidad que él en la casa , eso de llevar la casa... él no tiene idea por ejemplo de qué vamos a hacer de almuerzo, él no tiene idea de que hoy en día se lavó, ni que hay que lavar, ni que no sé falta verdura y hay que decirle a la nana "sabe que tiene que ir a comprar", no, yo siento que yo tengo en la casa más responsabilidad que él, pero eso es porque yo lo decidí así , porque yo en el fondo me gusta a mí llevar como la casa”. (Carla, 37 años)

Los roles que cumplen las mujeres en sus familias dan cuenta de mujeres con iniciativa, que lideran procesos y acciones en su realidad familiar. De alguna manera son ellas quienes orientan o guían gran parte de las 
tareas y decisiones cotidianas que se toman en el hogar, como sostiene un varón:

“Claro, por un tema de habilidades, mi señora tiene más habilidades con el tema de organizar cosas, yo soy más ejecutor [...] ella distribuye las tareas, ella distribuye y yo soy ejecutor” (Clemente, 32 años)

Más allá de la orientación femenina a sus parejas en la realización del trabajo reproductivo, la organización del trabajo productivo tiene grandes repercusiones en la toma de decisiones, división del trabajo doméstico y organización de la vida familiar.

\section{Cambios en los roles de género: búsqueda de complementariedad en la organización familiar}

Existen diversas estrategias a partir de las cuales las familias van resolviendo los requerimientos de la vida doméstica cotidiana y de las labores de crianza de los hijos. Los arreglos emergentes dan cuenta tanto de una división "democrática” de las tareas, como también de arreglos tradicionales en que se delega en manos femeninas una parte de las laborestradicionalmente ejecutadas por las mujeres.

Desde los discursos de las participantes del grupo de discusión se plantea que existe una complementariedad en la organización de la vida reproductiva, ya que existen ámbitos de responsabilización de cada uno de los miembros de la pareja. Esta complementariedad tiene ciertas particularidades, como la que pareciera ser la tendencia, que es que los hombres “eligen” o expresan ciertas preferencias por las tareas que desean realizar, mientras que la mujer va liderando los procesos, organizando y recibiendo apoyo de terceras -lo que da cuenta de la feminización del trabajo, y de la alta importancia que cobra la red de apoyo en la organización doméstica, en las que “otras” mujeres realizan aportes fundamentales.

La complementariedad en la realización de las tareas, se realiza en general dentro del marco de la orientación o liderazgo femenino, tal como se expresó en el punto anterior. Como señala una entrevistada, evidenciando la participación masculina a partir de su requerimiento:

“Ningún atado, o sea, en la casa él lava, tiende, puede hacer las camas, si uno se lo pide...o sea no... no tengo ningún problema. Siempre han sido bien compartidas las labores. (Paulina, 40 años)

Si bien esta disposición refleja mayor participación masculina, asimismo da cuenta de la discrecionalidad del varón para decidir cuándo y qué labores realizar, mostrando claramente la baja responsabilización por la vida reproductiva, evidenciando roles de género asimétricos.Una participante de un grupo de discusión, Jessica, señala que ella “es una mujer práctica, muy organizada”, haciéndose cargo de gran parte de las tareas, mientras su marido se encarga "del exterior del hogar", arreglo que encontramos en 
muchas de las entrevistadas.La complementariedad tiene un carácter diferente en aquellas parejas en que existen arreglos domésticos tendientes a la corresponsabilidad:

“...Nos levantamos, nos bañamos toda la cosa, y yo la voy a dejar al trabajo porque no sabe manejar yo sí, entonces ya, la voy a dejar al trabajo me devuelvo a la casa y ahí me quedo yo con Simón, que es mi hijo ya, eh, durante la mañana estamos los dos juntos, yo trabajo en esa jornada pero en la casa, tengo permiso de la universidad para avanzar en investigación, análisis de datos, esas cosas, entonces ahí voy jugando entre hacer el aseo, estar con él, hacer el, cachai, todos esos roles” (José, 29 años)

En este contexto, aparece como fundamental el proceso de toma de decisiones en la pareja, a través de conversaciones en las cuales la negociación es el principal mecanismo, como se desarrollará a continuación.

\title{
Negociación y toma de decisiones
}

Un hallazgo significativo se relaciona a la forma en que las familias de clase media en Concepción están tomando sus decisiones. En contraste con la visión tradicional donde la figura del padre aparece como la autoridad última y quien efectivamente decide por el grupo familiar (Olavarría, 2000), los relatos emergentes tanto en grupos de discusión como en las entrevistas realizadas apuntan hacia una negociación en términos de pareja, en un formato más democrático y relacional, cuestionándose mutuamente y valorándose los acuerdos por sobre las ideas individuales (Valdés, Caro et al., 2005)

\begin{abstract}
"siempre con la Patricia llevamos una súper buena relación y conversamos todas las cosas, obviamente hay, todas las parejas tienes sus conflictos menores pero todas las decisiones en la casa se toman en base a conversaciones, a discusiones en buena cachai, nunca se hace lo que yo quiero y si no cagaste, no... Siempre todas las conversaciones han tenido ese tenor y yo creo que por eso hemos durado tanto tiempo juntos también poh, nos complementamos bien, tenemos visiones distintas pero la mayoría de las veces convergemos en las mismas” (Marcelo, 32 años)
\end{abstract}

Uno de los elementos que consideramos como cruciales respecto de este cambio en torno a las prácticas de toma de decisiones, es la masiva salida de la mujer al mundo laboral, conformándose este elemento como central en su construcción identitaria y no como una actividad anexa o esporádica, configurándose desde este punto relaciones más paritarias y menos asimétricas (Todaro y Yañez, 2004, Valdés, Caro et al., 2005)

"Por ejemplo que ambos trabajemos tiene que ver con lo que cada uno contribuye económicamente en el hogar, porque eso... porque eso a la hora de tomar decisiones cuenta, entonces... o la división de 
los tiempos, de repente... no se poh, que yo a veces tuviera que quedarme a trabajar más del horario, pero... las decisiones... las grandes decisiones siempre las hemos tomado en conjunto y conversando. Ya las decisiones como más, no sé poh, más ordinarias, si se pueden llamar así, las toma él o las tomo yo, no... no influye mucho” (Paula, 29 años)

En términos cotidianos, como ya se esbozó, en la mayoría de los casos las decisiones de mantenimiento y funcionamiento del hogar son tomadas por la mujer, dedo queaun cuando existen estos cambios y se van configurando pautas emergentes de relaciones en la pareja, todavía subsisten importantes continuidades respecto del modelo tradicional masculino hegemónico (Olavarría, 2000; Valdés, Caro et al., 2005). Existe sí una amplia coincidencia entre los relatos obtenidos del trabajo de campo, en el sentido de que la pareja debe mantener una coherencia en la toma de decisiones respecto de lo presentado a sus hijos e hijas, entendidos éstos/as como el eje principal respecto al cual se construye y organiza la familia (Valdés, Caro et al., 2005) especialmente en el sentido valórico de la formación, configurándose una necesidad de no desautorizar al otro miembro de la pareja, aunque existan diferencias, las cuales deben ser conversadas a posteriori,

“Los dos, en este caso no se da el tema de que la mamá es la bruja y el papá cede, o al revés, que el papá es no sé qué y la mamá cede, y de hecho tenemos un acuerdo que ha funcionado rebien que es el tema de no, de nunca desautorizar al otro, porque esas cosas son las que van generando ruido y los niños naturalmente son manipuladores” (Fernanda, 39 años)

Las decisiones en la pareja se configuran entonces como un campo de negociación, no exento de conflictos, pero en donde existe una marcada búsqueda por los consensos y destacan las coincidencias por sobre las divergencias, en un contexto en donde las mujeres están cada vez más incorporadas al mercado laboral y los varones más interesados por aumentar su participación en las tareas de crianza (Valdés, Caro et al., 2005; Todaro y Yañez, 2004; Ranson, 2012; Brandth y Kvande, 2002; Valdés, 2009).

Una buena parte de las labores productivas y reproductivas se mantienen en el ámbito de competencia femenina, lo que implica la articulación de redes de apoyo para que la mujer pueda conciliar vida familiar y trabajo.

\section{Redes de apoyo y feminización de los cuidados}

Uno de los puntos relevados con mayor fuerza durante ungrupo de discusión, y que así mismo emergió también en las entrevistas realizadas, tiene que ver con la importancia de las distintas redes de apoyo para la crianza de los hijos e hijas y para el mantenimiento doméstico. En este sentido, emerge la figura de la familia más extensa, abuelos, hermanos, cuñados, hermanas, pero especialmente la abuela o la suegra, como elementos centrales de apoyo, respecto de los cuales se puede confiar a hijos o hijas 
y así los miembros de la pareja pueden realizar con tranquilidad actividades en las cuales no pueden participar sus hijos/as,

"había un tercer actor en ese caso que eran los abuelos, pero los abuelos retiraban a las niñas en la hora de almuerzo y la llevaban para su casa y ahí era donde recibían todos los días con un, un circo porque eran adoradas, entonces ahí ellas gozaban y todo el cuento y yo no las veía desde, muchas veces las veía al otro día en la mañana” (Javier, 50 años)

La otra figura que aparece constantemente en los relatos es la asesora del hogar, coloquialmente nombrada como nana, quien asume tareas de cuidado y crianza, así como mantenimiento del entorno doméstico, cuando padre y madre se encuentran fuera del hogar debido asus jornadas laborales. Existe una amplia diversidad de relatos en torno a las nanas, desde aquellas encargadas absolutamente de todas las tareas al interior del hogar hasta aquellas contratadas para específicamente tareas de cuidado de hijos e hijas, pero en todos los casos en donde está presente, se repite la importancia de este actor en el funcionamiento de las familias. Ello se evidencia en el relato de este padre participante de un grupo de discusión:

“Lo que yo quiero clarificar es que dije que pa’ nosotros su principal ayuda y labor es estar con Javiera y cuidarla pero la verdad es que igual, o sea, eh, cumple el papel más tradicional de nana, es decir, hace las cosas en la casa, qué se yo, vivimos en un departamento entonces tampoco es que le ocupe tanto tiempo, entonces, el principal tiempo, la mayor parte del tiempo es estar con la Javiera, pero igual claro, Javiera en general se despierta tarde, entonces en todo ese rato ella está haciendo como las cosas de la casa”. (Raimundo, 38 años)

En este punto es importante destacar la alta feminización de las redes de apoyo mencionadas en los discursos levantados, debido a que se tiende a considerar a las abuelas (madres y/o suegras)como personas de mayor confianza, así como frecuentemente la nanarefleja también la red femenina de apoyos, marcando una clara continuidad conlas prácticas tradicionales de llevar a cabo la crianza principalmente a partir del apoyo de otras mujeres (Valdés, Caro et al., 2005).

\section{Relaciones de género y redefinición de roles en la pareja}

Así, la redefinición de roles de género estaría desembocando en una mayor participación masculina en el mundo reproductivo, bajo un marco de complementariedad con sus parejas, quienes guían u orientan su participación. Lo anteriorincide en los cambios en los modelos de socialización de género de hijos e hijas.

"Sí poh, sí, yo igual les he enseñado que mucha diferencia entre hombre y mujer no debe haber más que la parte biológica como dices 
tú , porque si él tiene pena también tiene que llorar si es persona, que no solamente lloran las mujeres , también lloran los hombres, yo le he ido enseñando eso”. (Carla, 37 años)

Aun cuando se restringen, persisten los ámbitos de privilegio para los varones, donde se observa que el ocio constituiría un espacio en disputa, en el cual incluso el hombre corresponsable expresaríacon mayor claridad sus derechos que su pareja.

De la misma forma, se observan crecientes tensiones y disputas por arreglos domésticos más igualitarios, en parte vinculados al liderazgo femenino ya mencionado y, también, a la presión por una mayor responsabilización masculina (y no sólo la participación del varón en tareas específicas). Se busca un mayor equilibro en los roles de género, y aun cuando aparece en el discurso, éste diverge ampliamente de las prácticas. Ello va de la mano de la sobrevaloración del trabajo doméstico masculino y la subvaloración del trabajo femenino, aspecto que denota la persistencia de la asignación de género en el ámbito reproductivo.

\section{Otra mirada al ser hombres: paternidades e involucramiento creciente}

El rol delos hombres en el hogar ha mostrado cambios significativos durante las últimas décadas, tomando ciertos espacios más vinculados a la afectividad, y a las labores cotidianas de crianza. Este involucramiento va de la mano con la mayor receptividad y apertura de los varones a asumir estos temas; incluso encontramos algunos casos de reasignaciones de ámbitos de acción con sus esposas (al menos durante un periodo de tiempo). Se aprecian nuevos elementos constitutivos de identidad, como es el quedarse en casa con hijos. Como se ha planteado en la literatura, la infancia tiene un rol fundamental en reformular el "lugar que toma el padre", que cada vez "elige" tener mayor participaciónen la crianza de los hijos (Valdés, 2009).

Ello converge con lo planteado por Valdés (2009), respecto al declive de la autoridad paterna tradicional, y el surgimiento de nuevas representaciones sobre la paternidad -no obstante persisten ciertas resistencias a l variación de los roles que asume cada integrante de la pareja en el espacio doméstico, como ya se mencionó.El involucramiento en la crianza de hijos e hijas evidencia una responsabilidadcompartida. Uno de los entrevistados que temporalmente intercambió roles con su esposa, plantea:

“Tú tienes que como padre digamos, hacer esa pega, cambiar pañales y todo el cuento pero no solamente eso, no solamente la alimentación si no que hay una serie de ámbitos que se descubren...muchos más y con muchas complejidades pero con una riqueza inmensa, tremenda, o sea, yo veo...yo... es una experiencia cotidiana que yo la vivo pero yo me considero dentro de este grupo que te puedo...que describir de amigos, de gente con la que me relaciono en el trabajo qué sé yo, que...que tengo más habilidades que ellos... eso lo declaro 
digamos (risas), tengo cierta, cierta, o sea, más habilidades que ellos en términos de poder resolver los las cosas cotidianas, del hogar, estoy mucho más afín a eso...a y no tener un cuestionamiento de decir: "esas son pegas de mujer o estas no son pegas de hombre”, en fin...” (Javier, 50 años)

Asimismo, existen arreglos variables y flexibles en relación a cómo compartir el trabajo reproductivo, compatibilizándolo con la disponibilidad de tiempo. Se plantea, que esto ocurre aun cuando el marido llega tarde a la casa,

“... por ejemplo después que yo me acuesto yo no me levanto, si los niños necesitan algo mi marido es el que los ve, de hecho ellos solos lo tienen en su consciente porque si a ellos les pasa algo o quieren algo en la noche, llaman papá no llaman mamá. De esa forma nos organizamos, él se hace cargo de los niños de noche y yo todo el día... es súper buena. Una relación... por eso es que los niños llaman a él cuando quieren algo de noche, porque yo creo que de noche se sienten más protegidos por el papá que por mí, aunque de día ellos no son nada sin mí porque todo pasa por mí...” (Carla, 37 años)

Al mismo tiempo, la participación de los padres implica una mayor presencia como cuidadores de sus hijos e hijas, llegando a ser incluso los cuidadores principales:

"Lo voy a buscar y normalmente yo le preparo las comidas, eh no sé, lentejas, sopas, igual como que en ese sentido, eh, con el tema de las comidas yo igual juego un rol importante en el tema de que no sea mañoso, de ser como paciente, porque igual mi pareja es media impaciente con esas cosas, eh, por ejemplo cuando tuvo que aprender a tomar mamadera, igual yo ahí eh estuve dando eh estuve enseñándole igual, y después hubo harto tiempo que tomaba mamadera solo conmigo o las comidas igual hubo un tiempo que, que solo comía conmigo" (Néstor, 21 años)

Este mayor involucramiento en la crianza, se evidencia también en una relación más afectuosa con los hijos. Una entrevistada señala:

"Yo me enamoro de mi marido cuando veo la relación que tiene con mi hija, es una relación bonita, él está, ellos conversan, ella le cuenta cosas y eso para mí es importante porque en el fondo la figura paterna (da lo mismo si es el abuelo o el tío en familias donde no existe el papá) pero la figura paterna es importante sobre todo en las niñitas, es bonito verlo. (Fernanda, 39 años).

Si bien persisten importantes desafíos en términos de la división del trabajo doméstico y en términos de cómo la organización social de la vida productiva logra incorporar a los varones (Ranson, 2012), la puerta de entrada continúa siendo el involucramiento de los padres con la crianza de 
sus hijos, ya sea en actividades lúdicas o de cuidado. Esto se refleja en el discurso de un padre que participó en el grupo de discusión, quien tiene nacionalidad de origen europeo:

"No sé, yo creo que depende, en mi caso que yo trabajo al menos, como que, y, están los de ustedes, más igualitarios ¿no? Que hay, hay otra división de rol también, o que se justifica que uno a veces hace más por estar en la casa, con los niños, como, claro, no sé, hay que buscar el camino, lo que le gusta hacer a uno más, lo que puede hacer mejor y tratar de buscar el equilibrio en eso ¿no? Y tampoco diciendo como que, no sé, hay que romper con los roles tradicionales y la mujer no puede hacer nada de los roles tradicionales entonces la mujer no puede hacer nada porque está [risas] más bien aquí hay que compartirlo entre los dos, como que, alguien lo tiene que hacer, no como que, yo igual soy de eso, no sé, lo mejor sería que alguien esté con los niños, tal vez no todo el tiempo pero harto tiempo ¿no? Eso de, no sé, hacer los dos carrera al 100\% al final va a costa de los niños, siempre que la mujer tiene que quedar, antes ¿no? Que cuidaba a los niños y el marido llegaba y se le servía, quería salir con sus amigos, no sé cómo era eso pero, claro que sí, eso es importante, que las cosas se hacen, que uno pase tiempo con los niños, y, sea quien sea, pero que igual son cosas importantes aunque sean cosas tradicionales, deberían estar divididos los roles tradicionales yo creo” (Andrés, 37 años).

Los casos de Andrés y Néstor expuestos más arriba, dan cuenta de ruptura significativas, dado que son varones que han alternado la proveeduría con el rol de cuidador principal de sus hijos-considerando incluso el cuidado emocional de éstos. Ello muestra gran versatilidad en su rol paternal, y una muy alta valoración de su bienestar, en un sentido amplio.Un último aspectoa mencionar es la mayor consideración de la subjetividad de hijos e hijas, y a la vez, el hecho que estos experimentarían una socialización de género menos estereotipada, permeando de esta manera las relaciones de género y las dinámicas familiares.

\section{Discusión}

Los resultados de esta investigación indican que existe una gran diversidad en los arreglos domésticos existentes en los hogares del Concepción urbano, así como también distintas perspectivas respecto al rol que juegan los hombres en la crianza de las y los menores.Si bien persiste una división del trabajo relativamente "tradicional”, dado que parte significativa de las labores domésticas es realizada por mujeres -ya sean las madres-esposas, nanas, abuelas u otras familiares, se observan algunas rupturas en esta tendencia, con hombres crecientemente integrándose y responsabilizándose por las labores reproductivas. Este involucramiento incidiría en las configuraciones identitarias masculinas, que están siendo permeadas por las prácticas cotidianas de trabajo doméstico, cuidado y 
crianza de hijos e hijas, impactando las distintas formas en que se vive la paternidad. En este sentido, la paternidad adquiriría nuevos matices en la construcción de la identidad masculina donde el rol de proveedor sigue siendo su importancia, pero la cercanía afectiva y el involucramiento cotidiano en la crianza permearían las identidades masculinas emergentes.

En términos generales, si pensamos en una suerte de gradiente en los polos de desigualdad-igualdad de género, observamos tres ámbitos centrales en los cuales se ubican las relaciones familiares y los arreglos domésticos:

1) La mantención de ciertos ámbitos de privilegio masculinos y roles tradicionales de género -que implica que los varones aún no se responsabilizan por el trabajo doméstico, y a la vez “eligen” realizar las tareas domésticas de su preferencia.Esa participación tiende a ser más pasiva y mediada por la “orientación” femenina;

2) La emergencia de importantes espacios de disputa y múltiples fuentes de tensiones, negociaciones y conflictos. Existen diversos ámbitos en los que se observa una disputa por el poder: ello se observa en cómo se toman las decisiones, cómo se significa el trabajo de la mujer, qué ocurre con el liderazgo femenino que presiona la organización doméstica del hogar. Al mismo tiempo, está la tensión de compatibilizar trabajo-familia, presión que continúa siendo mayormente asumida por las mujeres; en algunos casos llevando a la reasignación de ámbitos de competencia de la vida reproductiva/ productiva.

3) Algunas rupturas, evidenciadas en algunos casos de corresponsabilidad parental, y en la reasignación de los ámbitos centrales de competencia, en los cuales el varón está fuertemente vinculado al ámbito reproductivo y la mujer centralmente al ámbito productivo.

Dicho lo anterior, este artículo aporta al campo de estudio de las relaciones de género en los arreglos domésticos urbanos evidenciando las transformaciones que se dan al interior de los mismos, donde si bien aún persiste lodoméstico como un ámbito feminizado -especialmente en términos de responsabilización femenina.Los casos de corresponsabilidad encontrados, o cercanos a ella, dan cuenta de rupturas significativas, especialmente en el caso de s parejas más jóvenes, con prácticas permeadas por un discurso vinculado a la corresponsabilidad y a la equidad de género, instalándose Significativamente en casi en prácticamente la totalidad de los casos de informantes de los hallazgos aquí expuestos, lo que también ha sido evidenciado por otras investigaciones en diversos contextos.

Se torna necesario, en nuevas investigaciones, explorar en mayor profundidad las brechas que pueden existir entre los discursos sobre articulación del mundo productivo y reproductivo, y las prácticas concretas que mujeres y hombres despliegan en este sentido, lo que se puede llevar a cabo mediante la triangulación de entrevistas con encuestas de uso de tiempotemporalmente Por otro lado, es necesario explorar las prácticas de 
socialización de género que se están generando a partir de la pluralidad de arreglos domésticos hallados en el mundo urbano, en especial en relación a los contrastes entre arreglos corresponsables y arreglos tradicionales, relevando cambios en las identidades de género y en la parentalidad.

\section{Agradecimientos}

Los resultados de investigación presentados en este artículo, se enmarcan en el Proyecto FONDECYT de Investigación 11130379 a mi cargo, financiado por la Comisión Nacional Científica y Tecnológica de Chile, CONICYT. Agradecimientos por sus comentarios a Cristián Jullian e Inti Fuica. 


\section{Bibliografía}

Aguayo, F., Correa, P., Cristi, P. (2011). Encuesta IMAGES Chile. Resultados de la Encuesta Internacional de Masculinidades y Equidad de Género. Santiago, Chile: CulturaSalud/EME.

Alméras, D. (2000). Procesos de Cambio en la Visión Masculina de las Responsabilidades Familiares. En J. Olavarría y R. Parrini (Eds.).Masculinidad/es. Identidad, Sexualidad y Familia. Primer Encuentro de Estudios de Masculinidad, pp. 91-102. Santiago, Chile: Flacso-Chile/Universidad Academia de Humanismo Cristiano/Red de Masculinidad.

Bourdieu, P. (2000). La dominaciónmasculina. Barcelona, España: ANAGRAMA.

Brandth, B. yKvande, E. (2002). Reflexive Fathers: Negotiating Parental Leave and Working Life.Gender, Work and Organization 9(2): 186203.

Campos, C. y Saldaña, L. (2018). 'Relaciones de género y arreglos en parejas de profesionales: ejecución v/s responsabilización’. EstudosFeministas 26(2).

Carrigan, T., Connell, B. y Lee, J. (1985). Toward a New Sociology of Masculinity.Theory and Society 14(5): 551-604.

Coltrane, S. (2010). Gender Theory and Household Labor.Sex Roles 63: 791-800.

Connell, R.W. (2005). Change among the Gatekeepers: Men, Masculinities, and Gender Equality in the Global Arena.Signs30(3): 1801-1825.

(1995). La organización social de la masculinidad. En J. Olavarría y T. Valdés (Eds.). (1997). Masculinidad/es. Poder y crisis. ISIS Internacional, Ediciones de las Mujeres $N^{\circ} 24$, Santiago.

Connell, R.W y J.W Masserschmidt. (2005). Hegemonic Masculinity: Rethinking the Concept.Gender and Society 19(6): 829-859.

Crompton, R. (2009). Gender inequality and the gendered division of labour. En J. Brown. (Ed.).The Future of Gender. Cambridge, UK: Cambridge University Press.

Craig, L., A. Powell y N. Cortis. (2012). Self-employment, Work-family time and the Gender Division of Labour.Work Employment Society 26(5): 716-734. 
Daly, M. (2011). What Adult Worker Model? A Critical Look at Recent Social Policy Reform in Europe from a Gender and Family Perspective. Social Politics 18(1): 1-23.

Deutsch, F. (2007). Undoing Gender.Gender and Society 21(1): 106-127.

Díaz, X., Godoy, L.yStecher, A. (2005). Significados del Trabajo, Identidad y Ciudadanía. La Experiencia de Hombres y Mujeres en un Mercado Laboral Flexible.Cuadernos de Investigación 3. Santiago, Chile: CEM.

Doucet, A. (2004). Fathers and the responsibility for children: A puzzle and a tension. Atlantis, 28 (2)

Elson, D. (1999). Labour Markets as Gendered Institutions: Equality, Efficiency and Empowerment Issue.WorldDevelopment 27(3), 611627.

Fuller, N. (1998). La constitución social de la identidad de género entre varones urbanos del Perú.En T. Valdés, y J. Olavarría (Eds.), Masculinidades y equidad de género en América Latina. Santiago, Chile: FLACSO.

García, B. y Oliveira, O. (2011). Cambios Familiares y Políticas Públicas en América Latina. Annual Review of Sociology(37), 613-633.

Gómez, V., y Jiménez, A. (2015). Corresponsabilidad familiar y el equilibrio trabajo familia: medios para mejorar la equidad de género. Polis (Santiago), 14(40), 377-396.

Gutmann, M. (2003).Changing Men and Masculinities in Latin America. Durham: Duke University Press.

Instituto Nacional de Estadísticas. (2016). Encuesta Nacional sobreuso del tiempo 2015. Santiago, Chile: Departamento de EstudiosSociales.

Marshall, K. (2006). Converging Gender Roles.Perspectives on Labour and Income 7(7): 5-17.

McDowell, L. (2004). Masculinity, Identity and Labour Market Change: Some Reflections on the Implication of Thinking Relationally about Difference and the Politics of Inclusion.GeografiskaAnnaler. Series B, Human Geography86(1): 45-56.

Méda, D. (2002).El tiempo de las mujeres. Conciliación entre vida familiar y profesional de hombres y mujeres. Narcea Ediciones.

OIT-PNUD. (2009).Trabajo y Familia: Hacia Nuevas Formas de Conciliación con Corresponsabilidad Social. Santiago, Chile: OIT-PNUD. 
Olavarría, J. (2005). ¿Dónde está el nuevo padre? Trabajo doméstico: de la retórica a la práctica. En X. Valdés y T. Valdés (Eds.) Familia y vida privada ¿Transformaciones, tensiones, resistencias o nuevos sentidos?, pp. 215-250.Santiago, Chile: FLACSO/CEDEM/UNFDA.

(2003). Men at Home? Child Rearing and Housekeeping among Chilean Working-Class Fathers. En M. Gutmann (Ed.).Changing men and masculinities in Latin America, pp. 333-347. Durham, EUA: DukeUniversityPress.

(2000). De la Identidad a la Política: Masculinidades y Políticas Públicas. Auge y Ocaso de la Familia Nuclear Patriarcal en el Siglo XX. En J. Olavarría y R. Parrini (Eds.) Masculinidad/es. Identidad, Sexualidad y Familia. Primer Encuentro de Estudios de Masculinidad, pp. 11-28. Santiago, Chile: Flacso-Chile/Universidad Academia de Humanismo Cristiano/Red de Masculinidad.

Olavarría, J yParrini, R. (Eds.) (2000).Masculinidad/es. Identidad, Sexualidad y Familia. Primer Encuentro de Estudios de Masculinidad. Santiago: Flacso-Chile/Universidad Academia de Humanismo Cristiano/ Red de Masculinidad.

PNUD. (2010).Desarrollo Humano en Chile. Género: los Desafíos de la Igualdad. Santiago, Chile: PNUD.

Ranson, G. (2012). Men, Paid Employment and Family Responsibilities: Conceptualizing the 'Working Father'.Gender, Work and Organization 19(6): 741-761.

Rehel, E. (2014). When Dad stays home too: Paternity leave, Gender and Parenting. Gender and Society 28(1): 110-132.

Ridgeway, C. yCorrell, S. (2004). Unpacking the Gender System: A Theoretical Perspective on Gender Beliefs and Social Relations.Gender and Society 18(4): 510-531.

Saldaña, L. (2009).Rural Labour in Neo-liberal Chile: Exploitation, Vulnerability and Cultural Transformation.Tesis de Doctorado no publicada (Colchester: Universidad de Essex).

Staab, S. (2012). Maternalism, Male-breadwinner Bias, and Market Reform: Historical Legacies and Current Reforms in Chilean Social Policy', Social Politics 19(3): 299-332.

Webb, J. (2009). Gender and Occupation in Market Economies: Change and Restructuring Since the 1980s.Social Politics 16: 82-110.

Todaro, R. y Yañez, S. (Eds.) (2004).El trabajo se Transforma. Relaciones de Producción y Relaciones de Género. Santiago, Chile: CEM ediciones. 
Valdés, X. (2009). El Lugar que Habita el Padre en Chile Contemporáneo. Estudio de las Representaciones sobre la Paternidad en Distintos Grupos Sociales.Polis 8(23): 385-410. https://scielo.conicyt.cl/ scielo.php?script=sci_arttext\&pid=S071865682009000200017\&lng=es\&thng=es

(2000). Masculinidad en el Mundo Rural: realidades que cambian, símbolos que permanecen. En J. Olavarría y R. Parrini (Eds.) Masculinidad/es. Identidad, Sexualidad y Familia. Primer Encuentro de Estudios de Masculinidad, pp. 29-46. Santiago, Chile: Flacso-Chile/ Universidad Academia de Humanismo Cristiano/Red de Masculinidad.

Valdés, X., Caro, P., Saavedra, R., Godoy, C., Rioja, T. y Raymond, E. (2005). Entre la reinvención y la tradición selectiva: familia, conyugalidad, parentalidad y sujeto en Santiago de Chile. En X. Valdés y T. Valdés (Eds.) Familia y vida privada ¿Transformaciones, tensiones, resistencias o nuevos sentidos?,pp. 163-214. Santiago, Chile: FLACSO/CEDEM/UNFDA.

Valdés X. y Valdés, T. (Eds.). (2005).Familia y vida privada ¿Transformaciones, tensiones, resistencias o nuevos sentidos? Santiago, Chile: FLACSO/CEDEM/UNFDA.

Valdés X. y Araujo, K. (1999).Vida Privada, Modernización Agraria y Modernidad. Santiago, Chile: CEDEM.

Viveros, M. (1998).Quebradores y cumplidores: biografías diversas de la masculinidad. En T. Valdésy J. Olavarría (Eds.), Masculinidades y equidad de género en América Latina. Santiago, Chile: FLACSO.

West, C. y Zimmerman, D. (1987). Doing Gender.Gender and Society 1(2): 125-151.

Recibido: 07.05 .18

Aceptado: 10.08 .18 